\title{
High rates of genome rearrangements and pathogenicity of Shigella spp.
}

\author{
Author names \\ Zaira Seferbekova ${ }^{1,2}$, Alexey Zabelkin ${ }^{3,4,5}$, Yulia Yakovleva, ${ }^{5,6}$, Robert Afasizhev², Natalia O. \\ Dranenko ${ }^{2}$, Nikita Alexeev ${ }^{3}$, Mikhail S. Gelfand ${ }^{2,7, \#}$, Olga O. Bochkareva ${ }^{2,8, \#}$ \\ Affiliation \\ ${ }^{1}$ Faculty of Bioengineering and Bioinformatics, Lomonosov Moscow State University, Moscow, \\ Russia \\ ${ }^{2}$ Institute for Information Transmission Problems (the Kharkevich Institute, RAS), Moscow, \\ Russia \\ ${ }^{3}$ Computer Technologies Laboratory, ITMO University, Saint-Petersburg, Russia \\ ${ }^{4}$ JetBrains Research, Saint-Petersburg, Russia \\ ${ }^{5}$ Bioinformatics Institute, Saint-Petersburg, Russia \\ ${ }^{6}$ Department of Cytology and Histology, Saint-Petersburg State University, Saint-Petersburg, \\ Russia \\ ${ }^{7}$ Skolkovo Institute of Science and Technology, Moscow, Russia \\ ${ }^{8}$ Institute of Science and Technology (IST Austria), Klosterneuburg, Austria
}

\section{Corresponding authors}

\# OOB: olga.bochkareva@ist.ac.at, MSG: mikhail.gelfand@iitp.ru

Keyword

Shigella, Escherichia coli, genomic rearrangements, pathogens, recombination, IS, E3 ubiquitinligases

\section{Abstract}

Shigella are pathogens originating within the Escherichia lineage but frequently classified as a separate genus. Shigella genomes contain numerous insertion sequences (ISs) that lead to pseudogenization of affected genes and an increase of non-homologous recombination. Here, we study 414 genomes of E. coli and Shigella strains to assess the contribution of genomic rearrangements to Shigella evolution. We found that Shigella experienced exceptionally high rates of intragenomic rearrangements and had a decreased rate of homologous recombination compared to pathogenic and non-pathogenic $E$. coli. The high rearrangement rate resulted in independent disruption of syntenic regions and parallel rearrangements in different Shigella lineages. Specifically, we identified two types of chromosomally encoded E3 ubiquitin-protein ligases acquired independently by all Shigella strains that also showed a high level of sequence conservation in the promoter and further in the 5 ' intergenic region. In the only available enteroinvasive $E$. coli (EIEC) strain, which is a pathogenic $E$. coli with a phenotype intermediate between Shigella and non-pathogenic $E$. coli, we found a rate of genome rearrangements comparable to those in other E. coli and no functional copies of the two Shigella-specific E3 ubiquitin ligases. These data indicate that accumulation of ISs influenced many aspects of genome evolution and played an important role in the evolution of intracellular pathogens. Our research demonstrates the power of comparative genomics-based on synteny block composition and an important role of non-coding regions in the evolution of genomic islands. 


\section{Importance}

Pathogenic Escherichia coli strains frequently cause infections in humans. Many E. coli exist in nature and their ability to cause disease is fueled by their ability to incorporate novel genetic information by extensive horizontal gene transfer of plasmids and pathogenicity islands. The emergence of antibiotic-resistant Shigella spp., which are pathogenic forms of $E$. coli, coupled with the absence of an effective vaccine against them, highlights the importance of the continuing study of these pathogenic bacteria. Our study contributes to the understanding of genomic properties associated with molecular mechanisms underpinning the pathogenic nature of Shigella. We characterize the contribution of insertion sequences to the genome evolution of these intracellular pathogens and suggest a role of upstream regions of chromosomal ipaH genes in the Shigella pathogenesis. The methods of rearrangement analysis developed here are broadly applicable to the analysis of genotype-phenotype correlation in historically recently emerging bacterial pathogens.

\section{Introduction}

Escherichia coli is likely the best-studied organism, at least on the molecular level. It is widely used to study fundamental aspects of bacterial genomics and is the subject of extensive research as an important component of the normal gut microbiota of vertebrates, including humans. While most $E$. coli strains are harmless, a non-negligible fraction is pathogenic, causing such diseases as diarrhoea, urinary tract infection, or meningitis $(1,2)$. Another group of pathogens, Shigella, which causes a severe form of bacillary dysentery, evolved from E. coli (1, 3 ). Shigella spp. are polyphyletic relative to E. coli, but the genus name is maintained in part due to the medical tradition (3-5). Nevertheless, from an evolutionary perspective, Shigella is just a set of strains causing a specific disease within the broader $E$. coli phylogenetic group.

Shigella strains carry a large plasmid ( $p / N V)$ which is essential for virulence $(1,3,5-8)$. They also might be distinguished from $E$. coli by their nonmotility with the associated absence of decarboxylated lysine, and by various biochemical characteristics, such as inability to ferment lactose and mucate (3). One more E. coli pathovar, enteroinvasive E. coli (EIEC), generally exhibits the same pathogenic and biochemical features as Shigella, including invasiveness that provided by the pINV $(1,3,5-8)$. Such phenotypic similarity may be attributed to adaptation to similar environmental conditions as Shigella and EIEC spend most of their lifecycle inside eukaryotic cells, while most $E$. coli strains inhabit extracellular space. Thus, EIEC could represent either a Shigella 'prototype', which could be a precursor for a typical Shigella, or a distinct group of pathogenic E. coli that have adapted to an intracellular lifestyle but, unlike Shigella, have not lost the ability to live outside eukaryotic cells $(5,6,8)$.

Acquisition of the virulence plasmid enabling intracellular lifestyle was likely a key event of Shigella evolution that facilitated further adaptation. It may have incorporated a variety of events, such as point mutations, suppression of certain genes, deletion of anti-virulence genes, or acquisition of insertion sequences. On the other hand, the intracellular niche may have provided a more relaxed selective pressure due to abundant resources and a lack of competitors $(1,6)$ that, in conjunction with lower effective population size, would have decreased the negative selection rate (9) and caused substantial changes in its genome arrangement and composition. Shigella genomes feature loss or inactivation of many genes, which has been attributed to the relaxation of selection acting on those genes (1). Gene deletions may also contribute to the specialization of bacteria and enable rapid adaptation to different conditions in the host cell (6, 10-12).

The chromosome and plasmids of Shigella species contain many insertion sequences (ISs), small mobile DNA fragments that easily translocate within the genome. An analysis of draft Shigella genomes demonstrated convergent loss of metabolic pathways by the integration of diverse ISs and pseudogenization by point mutations, often leading to degradation of multiple genes in the same pathway (13). However, the impact of IS elements on genome rearrangements and chromosome evolution has not been studied due to limitations arising from 
the use of incompletely assembled genome drafts. Indeed, the same repeated IS elements that increase the chromosome instability yield difficulties for the genomes assembly $(14,15)$.

In turn, repeats accumulation leads to genome rearrangements and changes in the expression of adjacent genes $(6,10,16-19)$ affecting the bacterial phenotype. The type of rearrangement depends on the mutual arrangement of the repetitive elements that have been involved in the recombination event. Recombination between inverted repeats leads to inversions, recombination between direct repeats leads to deletions, and recombination between direct repeats during replication leads to duplication (18). Since large deletions, insertions, and duplications are often under negative selection and are rare, inversions are the main drivers of structural changes in bacterial chromosomes (12). The frequency of rearrangements varies and may correlate with the presence of mobile genetic elements $(12,18)$.

Here, we provide a comprehensive analysis of the complete genomes of $E$. coli and Shigella strains based on the construction of synteny blocks and assess the contribution of insertion sequences to Shigella genome evolution. We show that Shigella genomes experienced exceptionally high rates of intragenomic rearrangements and a decreased rate of homologous recombination in comparison to other, in particular, pathogenic E. coli strains. Then we focus on rates of expansion of different ISs families and the patterns of their integration in genomes. Finally, we describe genome rearrangements that have occurred independently in separate lineages, showing convergent evolution of Shigella.

\section{Methods}

\section{Genomes}

We used all complete and annotated genomes of Shigella and E. coli available in GenBank as of April 2019 (11). We constructed a phylogenetic tree for E. coli strains only (see Methods below) and excluded clones and closely related E. coli strains from further analysis to reduce the size of datasets with minimal loss of diversity (Supplementary Figure S1a). Thus, for E. coli strains with identical names on short branches, we selected a random one and removed all others. In particular, we used only one reference genome for E. coli K12, E. coli O157:H7, E. coli O104:H4, E. coli O145:H28, E. coli BH100, E. coli Ecol, E. coli O127:H6, E. coli O25b:H4, E. coli O55:H7, E. coli ST540, E. coli ST2747, E. coli BL21/DE3, E. coli Nissle 1917, E. coli clone D i2, E. coli MRSN and E. coli AR strains (the list of excluded genomes is available on GitHub: https://github.com/zseferbekova/ShigellaProject/1Tree/Data/excluded_str ains.CSV). In total, we analysed 414 complete genomes, including 35 Shigella spp., 41 STEC, 31 ExPEC, 8 APEC, 7 ETEC, 3 EPEC, 3 AIEC, 2 EAEC, and 1 EIEC genome (Supplementary Table S1).

\section{Phylogenetic tree}

For the construction of the strains' phylogenetic tree, we used 238 universal, single-copy orthologous groups found in all 414 genomes. Orthologous groups were constructed using Proteinortho V5.13 (20) with parameters cov $=67$ (at least $67 \%$ coverage of both proteins in the BLAST alignment) and identity $=50$ (at least $50 \%$ identity in the common segments). Then we constructed a nucleotide multiple sequence alignment of genes in each orthologous group using Mafft (21) in the linsi mode. We then used RAxML (22) with the GTR+Gamma model and 100 bootstrap replicates to construct a phylogenetic tree based on the concatenated alignment of these genes (Figure 1). Finally, we used the GGRaSP (23) R package to divide all strains in the tree into seven clusters corresponding to the standard phylogroups (24). All trees were visualized using online iTOL (25).

Additionally we checked the robustness of the phylogenetic tree with regards to the identity threshold in the definition of orthologs. For that, we reclustered the orthologs with the protein identity threshold $=95 \%$ and compared the phylogenetic trees (Figure 1, Supplementary Figure S1b). The trees are consistent except for a small number of internal nodes with low bootstrap support. As we do not directly use the fine topology of the phylogenetic tree, that does 
bioRxiv preprint doi: https://doi.org/10.1101/2020.06.12.147751; this version posted March 17, 2021. The copyright holder for this preprint (which was not certified by peer review) is the author/funder, who has granted bioRxiv a license to display the preprint in perpetuity. It is made available under aCC-BY-NC-ND 4.0 International license.

not affect the results, nor does that influence the chromosome rearrangement analysis, as the latter has been performed directly on nucleotide sequences of the genomes.

\section{IS elements}

Online tool ISsaga (26) was used for annotation of IS elements in the chromosome sequences. For general statistical calculations, we used the number of predicted ORFs associated with ISs. For more precise annotation of ISs from different families, we used the number of predicted ISs that could include several ORFs.

\section{Synteny blocks}

The multiple whole-genome alignment for construction of locally-collinear blocks was performed with Sibeliaz (27). This new approach was specifically designed to address the performance issues caused by the large number of assembled genomes. It allowed us to scale our analysis to more than 400 genomes while maintaining accuracy. The k-mer size $(-k)$ was set to 15 , which is recommended by the documentation for bacterial genomes. Next, submodule maf2synteny from Ragout (28) was used to merge locally collinear blocks into synteny blocks (Supplementary Table S2). This approach is not sensitive to the annotation of genomes and identification of orthologs since it is based on compacted de Bruijn graphs constructed directly for nucleotide genomic sequences. The minimal block size $(-b)$ parameter was set to 1000 , the simplification parameter $(-s)$ was set to $f$ ine in order to retain the information about small-scale rearrangements. These stringent parameters allowed us to extend the analysis of rearrangements to recent pseudogenes, RNA genes, and conserved intergenic regions. Location of the synteny blocks in the chromosomes was visualised using Circos (29).

To infer the number of inversions on the phylogenetic tree we used common single-copy synteny blocks with the block size threshold $(-\mathrm{b})$ of $5 \mathrm{~kb}$. In each phylogenetic cluster, we used distance matrices where each element is a number of synteny blocks between given strains. We constructed trees based on the obtained matrices using PHYLIP (30) and the neighbour-joining algorithm.

\section{Inversions scenario}

We reconstructed the history of inversion events using MGRA (31). This tool takes as an input a phylogenetic tree and genomes represented as sets of synteny blocks. This analysis included chromosomes of all Shigella, related E. coli and several representative $E$. coli from each cluster not containing Shigella (51 genomes in total).

\section{Parallel rearrangements}

We say that a rearrangement is consistent with a tree if we may associate it with a particular branch on a tree, otherwise, we call a rearrangement parallel. We test each rearrangement for consistency with a tree with the standard Fitch algorithm (32). This approach allows us to detect the events occurring multiple times in distant clades, in particular, in different Shigella lineages.

To analyse inversions, we considered common single-copy blocks. For these 377 blocks, we constructed the breakpoint graph $(33,34)$ as follows. The graph is built on $377 \times 2$ vertices. For each block $B$, we introduce two vertices $B_{H}$ and $B_{T}$, its head and tail, respectively. If two blocks $B$ and $C$ are adjacent in genome $g$, the vertices $B_{\mathrm{H}}$ and $C_{\mathrm{T}}$ are linked by an edge of colour $g$. We note that since we consider only common blocks and all genomes are circular, the edges of each colour form a perfect matching on the graph vertices. Since some adjacency edges of different colours may link the same pair of vertices, we introduce multi-edges - a multi-edge is a set of parallel edges of different colours. The breakpoint graph for our data contains 754 vertices and 656 multi-edges. If two strains differ from each other by one inversion, this corresponds to a 4- 
bioRxiv preprint doi: https://doi.org/10.1101/2020.06.12.147751; this version posted March 17,2021 . The copyright holder for this preprint (which was not certified by peer review) is the author/funder, who has granted bioRxiv a license to display the preprint in perpetuity. It is made available under aCC-BY-NC-ND 4.0 International license.

cycle in the breakpoint graph (Supplementary Figure S2ab). For each multi-edge, we split the set of strains into patterns depending on the presence of the corresponding colour edge in the multiedge (Supplementary Figure S2c). Thus we associated each breakpoint (and each inversion) with a partition of the set of strains into patterns.

To analyse insertions, deletions, and duplications, we considered all blocks which were present in different copy numbers in some strains. For each block, we split the set of strains into patterns so that the copy number of this block in each pattern was the same (i.e. the pattern with strains containing 0 copies of the block, the pattern with strains containing 1 copy of the block, etc). Thus, we associated each copy number variation with a partition of the set of strains into patterns. Then, for blocks whose copy numbers differ in Shigella and E. coli, we manually classified the evolutionary scenarios based on the occurrence pattern and functional annotation of genes found in the block.

The developed pipeline for the detection of parallel rearrangements is available on GitHub: https://github.com/ctlab/parallel-rearrangements.

\section{Rates of homologous recombination}

For this analysis, we considered genomes from phylogroup B1 as it contains most of the Shigella strains and for better resolution used pairwise full-genome alignments constructed using MAUVE (35). Then for fragments without gaps, we calculated the number of non-identical columns in each $1 \mathrm{~kb}$ segment. Thus, for each pair of genomes, we constructed the distributions of the number of mutations across the genomes.

In the case of strictly vertical inheritance, this distribution would be Poisson with the parameter $\lambda$ reflecting the time of strain divergence. Fragments transferred horizontally from distant strains would contain more mutations yielding deviation from the Poisson distribution in the form of a heavy tail (36). The latter, being a mixture of the Poisson distributions with unknown parameters may be fitted by the Erlang distribution.

We have used the Python SciPy module (37) to fit all pairwise distributions by the function $F_{\lambda, k, \mu, W}(x)=W \times \mathbf{P}_{\lambda}(x)+(1-W) \times \mathbf{E}_{k, \mu}(x)$, where $\mathbf{P}_{\lambda}(x)=e^{-\lambda} \lambda^{x} / x$ ! is the Poisson distribution with parameter $\lambda, \mathbf{E}_{k, \mu}(x)=(x / \mu)^{k-1} e^{-x / \mu} /(\mu(k-1) !)$ is the Erlang distribution with the shape $k$ and scale $\mu$ (mode $=(k-1) \mu$, mean $=k \mu$, variance $\left.=k \mu^{2}\right)$, and the weight $W$ in the range $[0,1]$ measures the vertically inherited fraction of genome while $(1-W)$ corresponds to horizontally transferred fraction.

This approach extends the one suggested in (36). It averages over all genome segments, and hence is more robust than the approaches based on explicit identification of recombined segments, as the latter are sensitive to uneven evolutionary rates and, moreover, are computationally prohibitive for large-scale analyses. The Poisson parameter $\lambda$ monotonically increases with the divergence time of the vertically inherited genome fraction, and selecting pairs with the same $\lambda$, we obtain a set of strain pairs that have diverged at approximately the same time.

\section{Results}

\section{Structure of the phylogenetic tree and accumulation of IS elements}

We found 238 universal single-copy orthologous groups in 414 genomes (Supplementary Table S1) and used them to construct the unrooted phylogenetic tree (Fig. 1). The structure of the obtained phylogenetic tree recapitulates known E. coli phylogroups and supports the hypothesis that Shigella spp. included in our analysis evolved several times independently from E. coli and are named in accordance with the tree branches $(4,24)$. One of the Shigella genomes (GenBankID: GCA_001596115.1) was unclassified and did not cluster with any described Shigella species. Moreover, the source of the sample was lichen, which is highly unusual and unlikely for Shigella. Thus, we assumed that in this case the taxonomic annotation was wrong and did not consider this genome as Shigella. The only complete and annotated EIEC strain did not cluster with any Shigella (38). Other pathogenic E. coli strains also did not form any 
bioRxiv preprint doi: https://doi.org/10.1101/2020.06.12.147751; this version posted March 17,2021 . The copyright holder for this preprint (which was not certified by peer review) is the author/funder, who has granted bioRxiv a license to display the preprint in perpetuity. It is made available under aCC-BY-NC-ND 4.0 International license.

monophyletic clusters.

Shigella genomes generally encode more IS elements than non-pathogenic E. coli (10). To estimate the density of IS elements, we calculated their number in the chromosomes of all 414 strains (Fig. 1). The number of IS elements in Shigella genomes was significantly higher than in the genomes of pathogenic and non-pathogenic E. coli strains (Fig. 2a; the Wilcoxon-MannWhitney test, $p$-value $=2.4 \times 10^{-21}$ and $7.8 \times 10^{-18}$, respectively). Interestingly, the two most sequenced pathovars, STEC and ExPEC, had, respectively, significantly higher $\left(p=1.9 \times 10^{-4}\right)$ and lower $\left(p=3.5 \times 10^{-4}\right)$ number of IS elements than the average in non-pathogenic $E$. coli (Fig. $2 b)$.

To test whether the distribution of IS families differed in two clusters of Shigella we merged IS elements into larger families and mapped the results on the phylogenetic tree (Fig. 3 , 4). We found four IS families (IS1, IS3, IS4, IS91) which were enriched in all Shigella in comparison to E. coli. Moreover, we detected IS families that were specific for some Shigella lineages (IS21, IS110, IS630, IS66). However, as our dataset included only three S. boydii and two $S$. dysenteriae genomes, the results for these lineages should be considered as preliminary. By contrast, the $S$. sonnei group was represented by 11 strains and showed a significantly higher number of IS21, IS110 and IS630 elements (the Wilcoxon-Mann-Whitney test, $p=1.3 \times 10^{-8}$, $5.1 \times 10^{-7}, 1.9 \times 10^{-9}$, respectively).

\section{Composition of synteny blocks}

In all studied Shigella and E. coli genomes we found 4019 synteny blocks. The distribution of the synteny blocks by the number of strains in which they are present has an asymmetric Ushaped form similar to the distribution of gene frequencies in a population, also known as the $U$ curve (Fig. 5a). Only 377 synteny blocks were classified as universal, so that each block was found exactly once in all considered genomes, while other 3642 synteny blocks were not found, or found more than once, in at least one genome. The mean fraction of a chromosome covered by synteny blocks with the length threshold $1 \mathrm{~kb}$ was $62 \%$. The universal blocks spanned only $25-29 \%$ of the chromosome length and the distribution of these blocks across the chromosomes was not uniform, with long sections not harbouring any universal blocks (Supplementary Figure S3). The comparison of the distributions of common blocks across the chromosomes in different Shigella lineages, combined with GC-skew plots, revealed numerous unbalanced genomic rearrangements.

Most of the blocks are shorter than $5 \mathrm{~kb}$; some exceptionally long blocks are found among both common blocks and blocks that are present only in few strains (Fig. 5a). Common long blocks are formed by operons of housekeeping genes, the longest ones being (1) 20 genes including DNA polymerase III subunit alpha, components of the complex involved in the assembly of outer membrane proteins and elongation factors; (2) 19 genes including components of the cell division complex and the mur operon; (3) 14 genes that are components of the NADH-ubiquinone oxidoreductase complex. The longest rare blocks are formed by recent insertions such as prophages and pathogenicity islands.

The focus on the presence/absence patterns of synteny blocks allowed us to distinguish between genome rearrangements not affecting copy numbers (such as inversions) and those leading to copy number variations (such as insertions, deletions, and duplications). We then separately constructed the breakpoint graph for universal blocks and analysed phyletic patterns of non-universal blocks (see Methods). Thus we identified genetic features that could not be parsimoniously explained by common ancestry.

\section{Rates of genome rearrangements}

Shigella genomes are thought to be dynamic due to numerous IS elements that promote non-homologous recombination $(6,16,17)$. Thus, the rate of rearrangement in Shigella genomes may be higher in proportion to the accumulation of single nucleotide substitutions in comparison to non-invasive pathogenic E. coli. To test this possibility we compared the number of syntenic blocks with the number of single nucleotide substitutions in the universal genes for pairs of genomes in each phylogroup. The number of common single-copy synteny blocks between two 
bioRxiv preprint doi: https://doi.org/10.1101/2020.06.12.147751; this version posted March 17,2021 . The copyright holder for this preprint (which was not certified by peer review) is the author/funder, who has granted bioRxiv a license to display the preprint in perpetuity. It is made available under aCC-BY-NC-ND 4.0 International license.

genomes was inversely proportional to collinearity of the genomes, i.e. more collinear genomes had fewer blocks while more blocks corresponded to genomes with a large number of inversions. While the number of syntenic blocks may not accurately reflect the number of rearrangement events, especially when the number of such events is substantial, our approach provides a lower bound estimate of the number of inversion events. We then plotted the number of syntenic blocks relative to the number of single nucleotide substitutions for each pair of genomes (Supplementary Figures S3, S4).

Indeed, within the same interval of evolutionary distances between strains, in pairs of Shigella and $E$. coli strains ratio of a number of syntenic blocks to single nucleotide substitutions was substantially higher, in comparison to pairs of E. coli strains (the Wilcoxon-Mann-Whitney test, $p=2.22 \times 10^{-16}$ ) (Fig. 6a). Moreover, for pairs of Shigella this ratio is even higher and different in four Shigella lineages (Fig. 6b). Thus, genome rearrangements were occurring more frequently in the Shigella history compared to E. coli. In other pathogenic E. coli ratio of a number of syntenic blocks to single nucleotide substitutions did not differ from the average in nonpathogenic strains (Supplementary Figure S4a-d). The unclassified Shigella strain from lichen did not differ from E. coli strains (Supplementary Figure S4a), further supporting our assumption that this strain has been misclassified.

\section{Parallel genome rearrangements}

Events that occur multiple times independently on a phylogeny, called homoplasies, could indicate selection pressure acting on populations adapting to an intracellular lifestyle. Here we focus on events that have occurred several times independently in the Shigella lineages.

\section{Inversions and rearrangement hotspots}

For the universal synteny blocks, we found 25 parallel inversions and 40 rearrangement hotspots where a region had been involved in inversions several times independently (Supplementary Table S3). Some of these observations were easily explained by large regions between common single-copy synteny blocks that did not allow for an accurate reconstruction of the rearrangement breakpoints (Fig. 5b). For other events, we detected the disruption of synteny by insertion sequences that had been independently integrated into the same locus and then involved in different rearrangements. For instance, independent disruption of the regions between the pst operon (high-affinity phosphate transport system) and the atp operon (protontranslocating ATPase) occurred in four independent branches and participated in four different inversions (Fig. 7). Focusing on regions that were strongly syntenic in E. coli but involved in rearrangements in Shigella, we found two adjacencies that had been disrupted independently in S. sonnei, S. dysenteriae and S. flexneri. One more interesting event is the independent inversion of the $\mathrm{Na}+/ \mathrm{H}+$ antiporter gene in $\mathrm{S}$. sonnei and $\mathrm{S}$. flexneri lineages.

We reconstructed the history of inversions in 51 strains. This dataset includes all Shigella spp., closely related E. coli, and several representative E. coli from each cluster without Shigella (Supplementary Figure S5). Of 114 reconstructed inversion events, 103 were mapped to Shigella branches, while only 11 corresponded to $E$. coli branches. Of these inversions, 33 were mapped to the branch separating two $S$. dysenteriae; three other Shigella species demonstrated rearrangements at internal and terminal branches. These results are consistent with the estimation of rearrangement rates using the number of syntenic regions as the indicator.

\section{Deletions}

In addition to IS accumulation, Shigella adaptation had been accompanied by massive pseudogenization that in total resulted in genome reduction (39). These trends are well-known features of many pathogenic and symbiotic bacteria (40). Indeed, Shigella have significantly smaller genome size than all pathogenic and non-pathogenic E. coli (Supplementary Figure S6a). Note that the genome size of the two most abundant E. coli pathovars (ExPEC and STEC) is larger than that of non-pathogenic E. coli (Supplementary Figure S6b,c). Taking into account the high rate of non-homologous recombination, we anticipated seeing an increased rate of loss of non-universal synteny blocks in Shigella. We identified parallel insertions, deletions, and duplications in 2256 out of 3642 non-universal synteny blocks across the E. coli / Shigella 
bioRxiv preprint doi: https://doi.org/10.1101/2020.06.12.147751; this version posted March 17,2021 . The copyright holder for this preprint (which was not certified by peer review) is the author/funder, who has granted bioRxiv a license to display the preprint in perpetuity. It is made available under aCC-BY-NC-ND 4.0 International license.

phylogenetic tree (Supplementary Table S4). Three blocks lost in all Shigella and only two E. coli had affected the propionate catabolism (prpABCDER) operon. However, we did not find any strictly Shigella-specific large-scale deletion events.

\section{Insertions}

In contrast, we found one single-copy synteny block that was present in Shigella and EIEC but absent in other E. coli, likely indicating acquisition of this block in Shigella rather than multiple independent losses in E. coli. This region contained the gene IpaH1880 encoding an E3 ubiquitin-protein ligase, one of Shigella invasion-plasmid antigens, with a highly conserved 270 bp upstream non-coding region (Fig. 8a). In S. sonnei, S. flexneri, and EIEC the fragment was integrated in the same locus, while in $S$. boydii and $S$. dysenteriae this fragment was found in other loci (Supplementary Table S5). Although the ipaH genes are often surrounded by prophage genes and insertion sequences, they do not form stable genomic islands. Thus, the mechanism of $i p a H$ integration in chromosomes is uncertain.

One more block was present only in Shigella but absent in other E. coli including EIEC. This region also contained the genes ipaH0887/ipaH2610 encoding another E3 ubiquitin-protein ligase, again preceded by a conserved non-coding region of $230 \mathrm{nt}$ (Fig. 8b). In the chromosomes of $S$. flexneri we identified two copies of this block, located at a substantial distance from each other; in some strains, one of the gene copies is marked as a pseudogene. There are two possible evolutionary scenarios explaining this block duplication. One is gene duplication in the common ancestor of $S$. flexneri; another explanation is the independent acquisition of the copies by horizontal transfer. Based on adjacent blocks, the fragments in different strains cluster into two groups; $S$. sonnei and $S$. dysenteriae have their only copy in the first locus, while $S$. boydii has its only copy in the second locus (Supplementary Table S5). This pattern may be explained by the independent transfer of the genes via site-specific insertion or via homologous recombination as these mechanisms retain the gene environment.

The evolutionary history of syntenic blocks seems relevant to the functional specificity of Shigella and EIEC, as the products of $I p a H$ genes are secreted by intracellular bacteria via the type III secretion system (T3SS) (40) and, therefore, the IpaH genes repertoire may confer Shigella- or EIEC-specific functionality. Sequence conservation of the non-coding region indicates the importance of this sequence either for the integration or for the regulation of the $i p a H$ transcription. Being highly-conserved in different Shigella strains, these non-coding fragments are found only in $\mathrm{ipaH}$ upstream regions and are not homologous for two different ipaH genes. Thus, we tentatively suggest that these non-coding fragments hold regulatory elements and play a role in Shigella pathogenicity.

\section{Rates of homologous recombination}

We hypothesized that disruption of syntenic regions should decrease the rate of homologous recombination. To check this, we calculated fractions of horizontally transferred fragments in strains using pairwise genome alignments of E. coli, S. flexneri, S. boydii, and S. sonnei (see Methods). Indeed, at the same level of divergence between strains (with the Poisson $\lambda$ parameter in the vertically inherited fraction ranging from 0 to 1.45), pairs of Shigella strains had a significantly lower fraction of fragments horizontally transferred by homologous recombination, $(1-W)=0.094 \pm 0.017$, in comparison to pairs of $E$. coli strains, $(1-W)=0.612 \pm 0.002\left(p=4.17 \times 10^{-124}\right.$, the Wilcoxon test) (Fig. 9).

The estimated parameter $\lambda$ may reflect many evolutionary parameters such as the generation time and the intensity of selection. However, the observation that larger Poisson lambda corresponds to lower clonal fraction of the genome, as expected, demonstrates the consistency of our results. We used this technique for Escherichia pairs and Shigella pairs at the same level of sequence similarity, and hence demonstrated that the clonal genome fraction is larger in the latter, confirming our hypothesis. 
bioRxiv preprint doi: https://doi.org/10.1101/2020.06.12.147751; this version posted March 17,2021 . The copyright holder for this preprint (which was not certified by peer review) is the author/funder, who has granted bioRxiv a license to display the preprint in perpetuity. It is made available under aCC-BY-NC-ND 4.0 International license.

\section{Discussion}

Compared to other pathogenic E. coli, Shigella (i) accumulated a large variety of ISs, (ii) acquired new chromosomal genes, (iii) experienced exceptionally high rates of intragenomic rearrangements and (iv) had a decreased rate of homologous recombination.

The diversity of $E$. coli pathotypes is explained by the high plasticity of its genome, as genes responsible for pathogenic traits are mostly acquired by extensive horizontal gene transfer and are often conveyed by mobile genetic elements (41-43). Both Shigella and EIEC spend much of their life cycle within eukaryotic cells and share many invasion-related functional systems. The adaptation to an intracellular lifestyle was conferred by the acquisition of the pINV plasmid encoding a type III secretion system (T3SS) (40). Phylogenetic analysis supports the hypothesis that four existing Shigella lineages and the EIEC strain have arisen from different ancestral $E$. coli strains on multiple independent occasions $(3,4,7,8)$.

The delivery of bacterial virulence proteins, called "effectors," into host cells via T3SS plays a crucial role in the infection strategies of Shigella. Such effectors are involved in the reorganization of the host cell cytoskeleton and in the modulation of cellular signalling pathways that allow the bacteria to evade the host immune response (44). Each IpaH family protein likely has a specific host target protein due to the substrate recognition domain, and thus makes a distinct contribution to bacterial pathogenesis (40). Most T3SS effectors are encoded by plasmid pINV genes, while the biological role of chromosomally encoded ipaH genes remains obscure. Based on our results we hypothesize that some chromosomal E3 ubiquitin-protein ligases are essential for Shigella/EIEC pathogenicity while others may provide specific advantages. The differences in the composition of functional types of ubiquitin-protein ligases may also explain the differences in the infectious dose and disease severity between Shigella and EIEC pathotypes.

High numbers and the variety of mobile elements in evolutionarily young intracellular pathogens is usually explained by weaker selection against repetitive elements due to decreased effective population size $(9,17,38)$. IS elements may drive the evolution of chromosome organisation by causing non-homologous recombination (45). Genome rearrangements change the chromosomal architecture, which can alter gene composition and gene expression. These events are largely detrimental for free-living bacteria and are expected to be selected against (18). On the other hand, in a new environment, non-homologous recombination and the associated functional changes may provide a base for adaptation (46, 47). For instance, Burkholderia mallei, a young obligate intracellular pathogen, has lost numerous clusters of genes through IS-mediated elimination as demonstrated by the comparison of its genome to strains of the ancestral species, Burkholderia pseudomallei $(48,49)$. The genome reduction of $B$. mallei is up to $30 \%$ in some strains and the adaptation is still not complete (50). In comparison to other $E$. coli, Shigella have slightly smaller genomes and the evolution was accompanied by IS-mediated pseudogenization, but not large-scale deletions.

IS families differ in the expansion rate in Shigella lineages, which is expected given the independent origin of these lineages. Two types of IS (IS3, IS4) revealed high expansion rates in all Shigella lineages; this observation is in agreement with a recently published analysis of draft Shigella and EIEC genomes (13). These ISs are not common for E. coli populations but are a part of $p I N V$ that explains their expansion after the plasmid acquisition. In contrast, IS1 is present in many pathogenic $E$. coli and the difference in its frequency in pathogenic $E$. coli and $S$. sonnei is not statistically significant. The number of IS elements and, consequently, the rate of genome rearrangements in the EIEC strain were comparable with those in other E. coli. On the other hand, draft EIEC genomes featured larger IS frequencies in EIEC populations in comparison to other E. coli, but lower than in Shigella strains (13); but the rates of genomic rearrangements were not estimated in the cited paper as the studied genomes had not been assembled. Taken together, we propose that differences in the number of IS genomic elements may have influenced different stages of formation of intracellular pathogens EIEC and Shigella spp.

An expected consequence of frequent genome rearrangement is a decrease in the rate of 
homologous recombination. Indeed, in comparison to E. coli, Shigella genomes contain fewer DNA segments horizontally transferred by homologous recombination. However, this also could be explained by smaller population size and isolated intracellular lifestyle of Shigella strains. Homologous recombination levels in core genes, manifesting as incongruence of gene phylogenetic trees with the strain phylogeny, is smallest in endosymbionts and intracellular pathogens (51). These are likely interconnected processes. Bottlenecks and decreased selection pressure lead to the increase in the number of IS elements $(9,50)$; this in turn provides more opportunities for genome rearrangements that become tolerated due to decreased selection. Indeed, bursts of rearrangements were observed in the genomes of pathogens that had recently changed host and lifestyle, such as Yersinia pestis (48) and Burkholderia mallei (52). On the other hand, relative isolation of strains with the mainly intracellular lifestyle provides fewer opportunities for homologous recombination, while the lack of genome collinearity creates mechanistic obstacles to the process (53). At that, neither increased rearrangement rate, nor decreased homologous recombination rate are observed in intracellular EIEC strains of $E$. coli, supporting the link between these phenomena.

The analysis of genome rearrangements requires complete genomes, while less than $1 \%$ of available Shigella genomes have sufficient quality of assembly. Another issue is that misassemblies are mainly caused by genomic repeats and may be confused with true rearrangements. Although there are several strategies widely used for assembly validation such as long read (re)sequencing and/or PCR contiguity verification (54-56), some of the detected rearrangements may be caused by inaccuracies in gap closure procedures. While each particular observation requires experimental verification, the observed correlation between rearrangement rates and mutation rates in closely related strains allow us to conclude that assembly errors do not affect the evolutionary signal in the analysed data (52).

Due to high genomic plasticity, pathogenic E. coli are among the most frequent causes of bacterial infections in humans $(57,58)$. In particular, the E. coli O104:H4 outbreak in Germany in 2011 was caused by a strain that had acquired characteristics of two previously described pathotypes (59). The emergence of antibiotic-resistant Shigella with the absence of an effective vaccine highlights the importance of a detailed investigation of this pathogen (60). Our results contribute to the understanding of genomic properties associated with adaptation to intracellular lifestyle of Shigella and EIEC and the developed approaches should be broadly applicable to other young bacterial pathogens.

\section{Author statements}

\section{Authors and contributors}

MSG and OOB conceived the study, ZS, OOB, and MSG designed the study; ZS, RA, AZ, and NA developed the methods, ZS, YY, RA, ND and OOB analyzed the data; ZS, NA, OOB, and MSG wrote the manuscript. All authors read and approved the final version of the manuscript.

\section{Conflicts of interest}

The authors declare that they have no competing interests.

\section{Data summary}

The datasets supporting the conclusions of this article and used scripts are available at https://github.com/zseferbekova/ShigellaProject. The authors confirm all supporting data have been provided in the article or as supplementary data files.

\section{Funding information}

This study was supported by the Russian Science Foundation, grant 18-14-00358. The work of 
OOB is supported by the European Union's Horizon 2020 research and innovation programme under the Marie Skłodowska-Curie grant agreement No 754411. The work of NA is supported by the Government of the Russian Federation through the ITMO Fellowship and Professorship Program. The funding bodies had no role in the design of the study, collection, analysis, and interpretation of data and in writing the manuscript.

\section{Ethical approval}

Not applicable

\section{Consent for publication}

Not applicable

\section{Acknowledgements}

We thank Fyodor Kondrashov for valuable advice and manuscript proofreading. We also thank Alla Mikheenko for assistance with Circos.

\section{References}

1. The HC, Thanh DP, Holt KE, Thomson NR, Baker S. 2016. The genomic signatures of Shigella evolution, adaptation and geographical spread. Nat Rev Microbiol 14:235-250.

2. Zhang Y, Lin K. 2012. A phylogenomic analysis of Escherichia coli / Shigella group: implications of genomic features associated with pathogenicity and ecological adaptation. BMC Evol Biol 12:174.

3. Pupo GM, Lan R, Reeves PR. 2000. Multiple independent origins of Shigella clones of Escherichia coli and convergent evolution of many of their characteristics. Proceedings of the National Academy of Sciences.

4. Chaudhuri RR, Henderson IR. 2012. The evolution of the Escherichia coli phylogeny. Infect Genet Evol 12:214-226.

5. Lan R, Reeves PR. 2002. Escherichia coli in disguise: molecular origins of Shigella. Microbes Infect 4:1125-1132.

6. Beld MJC, Reubsaet FAG. 2012. Differentiation between Shigella, enteroinvasive Escherichia coli (EIEC) and noninvasive Escherichia coli. European Journal of Clinical Microbiology \& Infectious Diseases.

7. Yang J, Nie H, Chen L, Zhang X, Yang F, Xu X, Zhu Y, Yu J, Jin Q. 2007. Revisiting the Molecular Evolutionary History of Shigella spp. Journal of Molecular Evolution.

8. Lan R, Alles MC, Donohoe K, Martinez MB, Reeves PR. 2004. Molecular Evolutionary Relationships of Enteroinvasive Escherichia coli and Shigella spp. Infection and Immunity.

9. Mamirova L, Popadin K, Gelfand MS. 2007. Purifying selection in mitochondria, freeliving and obligate intracellular proteobacteria. BMC Evol Biol 7:1-12.

10. Yang F, Yang J, Zhang X, Chen L, Jiang Y, Yan Y, Tang X, Wang J, Xiong Z, Dong J, Xue Y, Zhu Y, Xu X, Sun L, Chen S, Nie H, Peng J, Xu J, Wang Y, Yuan Z, Wen Y, Yao Z, Shen Y, Qiang B, Hou Y, Yu J, Jin Q. 2005. Genome dynamics and diversity of Shigella species, the etiologic agents of bacillary dysentery. Nucleic Acids Res 33:6445-6458.

11. NCBI Resource Coordinators. 2018. Database resources of the National Center for Biotechnology Information. Nucleic Acids Res 46:D8-D13.

12. Achaz G, Coissac E, Netter P, Rocha EPC. 2003. Associations between inverted repeats and the structural evolution of bacterial genomes. Genetics 164:1279-1289.

13. Hawkey J, Monk JM, Billman-Jacobe H, Palsson B, Holt KE. 2020. Impact of insertion sequences on convergent evolution of Shigella species. PLoS Genet 16:e1008931.

14. Shapiro JA, von Sternberg R. 2005. Why repetitive DNA is essential to genome 
bioRxiv preprint doi: https://doi.org/10.1101/2020.06.12.147751; this version posted March 17,2021 . The copyright holder for this preprint (which was not certified by peer review) is the author/funder, who has granted bioRxiv a license to display the preprint in perpetuity. It is made available under aCC-BY-NC-ND 4.0 International license.

function. Biol Rev Camb Philos Soc 80:227-250.

15. Avershina E, Rudi K. 2015. Dominant short repeated sequences in bacterial genomes. Genomics 105:175-181.

16. Buchrieser C, Glaser P, Rusniok C, Nedjari H, D'Hauteville H, Kunst F, Sansonetti $P$, Parsot C. 2000. The virulence plasmid pWR100 and the repertoire of proteins secreted by the type III secretion apparatus of Shigella flexneri. Mol Microbiol 38:760-771.

17. Siguier P, Gourbeyre E, Chandler M. 2014. Bacterial insertion sequences: their genomic impact and diversity. FEMS Microbiol Rev 38:865-891.

18. Darling AE, Miklós I, Ragan MA. 2008. Dynamics of genome rearrangement in bacterial populations. PLoS Genet 4:e1000128.

19. Couturier E, Rocha EPC. 2006. Replication-associated gene dosage effects shape the genomes of fast-growing bacteria but only for transcription and translation genes. Mol Microbiol 59:1506-1518.

20. Lechner M, Findeiss S, Steiner L, Marz M, Stadler PF, Prohaska SJ. 2011. Proteinortho: detection of (co-)orthologs in large-scale analysis. BMC Bioinformatics 12:124.

21. Katoh K, Standley DM. 2013. MAFFT Multiple Sequence Alignment Software Version 7: Improvements in Performance and Usability. Molecular Biology and Evolution.

22. Stamatakis A. 2014. RAxML version 8: a tool for phylogenetic analysis and postanalysis of large phylogenies. Bioinformatics.

23. Clarke TH, Brinkac LM, Sutton G, Fouts DE. 2018. GGRaSP: a R-package for selecting representative genomes using Gaussian mixture models. Bioinformatics 34:3032-3034.

24. Dusek N, Hewitt AJ, Schmidt KN, Bergholz PW. 2018. Landscape-Scale Factors Affecting the Prevalence of Escherichia coli in Surface Soil Include Land Cover Type, Edge Interactions, and Soil pH. Appl Environ Microbiol 84.

25. Letunic I, Bork P. 2016. Interactive tree of life (iTOL) v3: an online tool for the display and annotation of phylogenetic and other trees. Nucleic Acids Research.

26. Varani AM, Siguier P, Gourbeyre E, Charneau V, Chandler M. 2011. ISsaga is an ensemble of web-based methods for high throughput identification and semi-automatic annotation of insertion sequences in prokaryotic genomes. Genome Biol 12:R30.

27. Minkin I, Medvedev P. 2020. Scalable multiple whole-genome alignment and locally collinear block construction with SibeliaZ.

28. Kolmogorov M, Armstrong J, Raney BJ, Streeter I, Dunn M, Yang F, Odom D, Flicek P, Keane TM, Thybert D, Paten B, Pham S. 2018. Chromosome assembly of large and complex genomes using multiple references. Genome Res 28:1720-1732.

29. Krzywinski M, Schein J, Birol I, Connors J, Gascoyne R, Horsman D, Jones SJ, Marra MA. 2009. Circos: an information aesthetic for comparative genomics. Genome Res 19:1639-1645.

30. Feisenstein J. 1989. PHYLIP: Phylogeny Inference Package Version 3.2 Manual.

31. Avdeyev P, Jiang S, Aganezov S, Hu F, Alekseyev MA. 2016. Reconstruction of Ancestral Genomes in Presence of Gene Gain and Loss. J Comput Biol 23:150-164.

32. Erdős PL, Székely LA. 1994. On weighted multiway cuts in trees. Math Program 65:93105.

33. Alekseyev MA, Pevzner PA. 2009. Breakpoint graphs and ancestral genome reconstructions. Genome Research.

34. Bafna V, Pevzner PA. Genome rearrangements and sorting by reversals, p. 148-157. In Proceedings of 1993 IEEE 34th Annual Foundations of Computer Science. IEEE.

35. Darling ACE, Mau B, Blattner FR, Perna NT. 2004. Mauve: multiple alignment of conserved genomic sequence with rearrangements. Genome Res 14:1394-1403.

36. Dixit PD, Pang TY, Studier FW, Maslov S. 2015. Recombinant transfer in the basic genome of Escherichia coli. Proc Natl Acad Sci U S A 112:9070-9075.

37. Virtanen $P$, Gommers R, Oliphant TE, Haberland M, Reddy T, Cournapeau D, Burovski E, Peterson P, Weckesser W, Bright J, van der Walt SJ, Brett M, Wilson J, Millman KJ, Mayorov N, Nelson ARJ, Jones E, Kern R, Larson E, Carey CJ, Polat İ, Feng Y, Moore EW, VanderPlas J, Laxalde D, Perktold J, Cimrman R, Henriksen I, Quintero EA, Harris CR, Archibald AM, Ribeiro AH, Pedregosa F, van Mulbregt P, SciPy 1.0 Contributors. 2020. SciPy 1.0: fundamental algorithms for scientific computing in Python. Nat 
Methods 17:261-272.

38. Bordenstein SR, Reznikoff WS. 2005. Mobile DNA in obligate intracellular bacteria.

Nature Reviews Microbiology.

39. Feng Y, Chen Z, Liu S-L. 2011. Gene decay in Shigella as an incipient stage of hostadaptation. PLoS One 6:e27754.

40. Ashida H, Toyotome T, Nagai T, Sasakawa C. 2007. Shigella chromosomal IpaH proteins are secreted via the type III secretion system and act as effectors. Mol Microbiol 63:680-693.

41. van Elsas JD, Semenov AV, Costa R, Trevors JT. 2010. Survival of Escherichia coli in the environment: fundamental and public health aspects. ISME J 5:173-183.

42. Touchon M, Hoede $\mathrm{C}$, Tenaillon $\mathrm{O}$, Barbe $\mathrm{V}$, Baeriswyl S, Bidet $\mathrm{P}$, Bingen $\mathrm{E}$, Bonacorsi S, Bouchier C, Bouvet O, Calteau A, Chiapello H, Clermont O, Cruveiller S, Danchin A, Diard M, Dossat C, Karoui ME, Frapy E, Garry L, Ghigo JM, Gilles AM, Johnson J, Le Bouguénec $C$, Lescat M, Mangenot S, Martinez-Jéhanne V, Matic I, Nassif X, Oztas S, Petit MA, Pichon C, Rouy Z, Ruf CS, Schneider D, Tourret J, Vacherie B, Vallenet D, Médigue C, Rocha EPC, Denamur E. 2009. Organised genome dynamics in the Escherichia coli species results in highly diverse adaptive paths. PLoS Genet 5:e1000344.

43. Dobrindt U, Chowdary MG, Krumbholz G, Hacker J. 2010. Genome dynamics and its impact on evolution of Escherichia coli. Med Microbiol Immunol 199:145-154.

44. Mattock E, Blocker AJ. 2017. How Do the Virulence Factors of Work Together to Cause Disease? Front Cell Infect Microbiol 7:64.

45. Raeside C, Gaffé J, Deatherage DE, Tenaillon O, Briska AM, Ptashkin RN, Cruveiller S, Médigue C, Lenski RE, Barrick JE, Schneider D. 2014. Large chromosomal rearrangements during a long-term evolution experiment with Escherichia coli. MBio 5:e01377-14.

46. Brandis G, Hughes D. 2020. The SNAP hypothesis: Chromosomal rearrangements could emerge from positive Selection during Niche Adaptation. PLoS Genet 16:e1008615.

47. Yan W, Wei S, Wang Q, Xiao X, Zeng Q, Jiao N, Zhang R. 2018. Genome Rearrangement Shapes Prochlorococcus Ecological Adaptation. Appl Environ Microbiol 84.

48. Bochkareva OO, Dranenko NO, Ocheredko ES, Kanevsky GM, Lozinsky YN, Khalaycheva VA, Artamonova II, Gelfand MS. 2018. Genome rearrangements and phylogeny reconstruction in. PeerJ 6:e4545.

49. Losada L, Ronning CM, DeShazer D, Woods D, Fedorova N, Kim HS, Shabalina SA, Pearson TR, Brinkac L, Tan P, Nandi T, Crabtree J, Badger J, Beckstrom-Sternberg S, Saqib M, Schutzer SE, Keim P, Nierman WC. 2010. Continuing evolution of Burkholderia mallei through genome reduction and large-scale rearrangements. Genome Biol Evol 2:102-116.

50. Mira A, Pushker R, Rodríguez-Valera F. 2006. The Neolithic revolution of bacterial genomes. Trends Microbiol 14:200-206.

51. González-Torres P, Rodríguez-Mateos F, Antón J, Gabaldón T. 2019. Impact of Homologous Recombination on the Evolution of Prokaryotic Core Genomes. MBio 10.

52. Bochkareva OO, Moroz EV, Davydov II, Gelfand MS. 2018. Genome rearrangements and selection in multi-chromosome bacteria Burkholderia spp. BMC Genomics 19:965.

53. Aguilera A, Rothstein R. 2007. Molecular Genetics of Recombination. Springer Science \& Business Media.

54. English AC, Richards S, Han Y, Wang M, Vee V, Qu J, Qin X, Muzny DM, Reid JG, Worley KC, Gibbs RA. 2012. Mind the gap: upgrading genomes with Pacific Biosciences RS long-read sequencing technology. PLoS One 7:e47768.

55. Madoui M-A, Engelen S, Cruaud C, Belser C, Bertrand L, Alberti A, Lemainque A, Wincker P, Aury J-M. 2015. Genome assembly using Nanopore-guided long and errorfree DNA reads. BMC Genomics 16:327.

56. Acuña-Amador L, Primot A, Cadieu E, Roulet A, Barloy-Hubler F. 2018. Genomic repeats, misassembly and reannotation: a case study with long-read resequencing of 
Porphyromonas gingivalis reference strains. BMC Genomics 19:54.

57. Allocati N, Masulli M, Alexeyev MF, Di Ilio C. 2013. Escherichia coli in Europe: an overview. Int J Environ Res Public Health 10:6235-6254.

58. Pasqua M, Michelacci V, Di Martino ML, Tozzoli R, Grossi M, Colonna B, Morabito S, Prosseda G. 2017. The Intriguing Evolutionary Journey of Enteroinvasive (EIEC) toward Pathogenicity. Front Microbiol 8:2390.

59. Burger R. 2012. EHEC O104:H4 IN GERMANY 2011: LARGE OUTBREAK OF BLOODY DIARRHEA AND HAEMOLYTIC URAEMIC SYNDROME BY SHIGA TOXINPRODUCING E. COLI VIA CONTAMINATED FOODImproving Food Safety Through a One Health Approach: Workshop Summary. National Academies Press (US).

60. Hassan R, Tantawy M, Gouda NA, Elzayat MG, Gabra S, Nabih A, Diab AA, El-Hadidi M, Bakry U, Shoeb MR, Elanany M, Shalaby L, Sayed AA. 2020. Genotypic characterization of multiple drug resistant Escherichia coli isolates from a pediatric cancer hospital in Egypt. Sci Rep 10:4165. 


\section{Figures and tables}

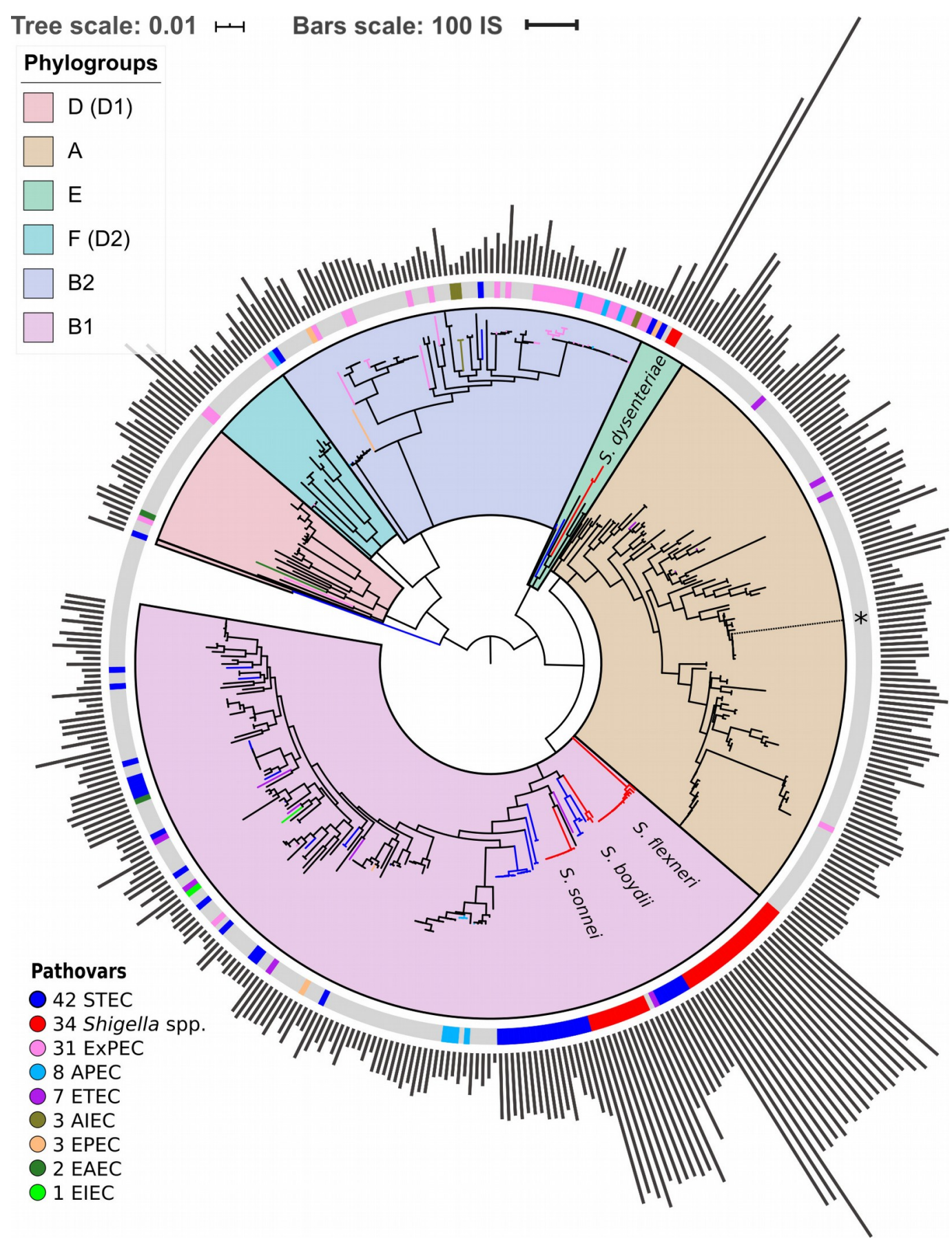

Figure 1. Phylogenetic tree of E. coli and Shigella spp. The tree is based on the nucleotide alignment of universal single-copy orthologs. Clusters and corresponding phylogroups are shown in different colours; the number of genomes of each pathovar is indicated to the right of the pathovar's name. Pathogenic E. coli strains are marked with coloured bands on the outer circle. The location of the unclassified Shigella strain is shown with an asterisk. The bars indicate the number of ISs found by ISsaga in bacterial chromosomes. The tree is unrooted. The tree is also available online (iTOL): https://itol.embl.de/tree/8589127100415001556065531. 

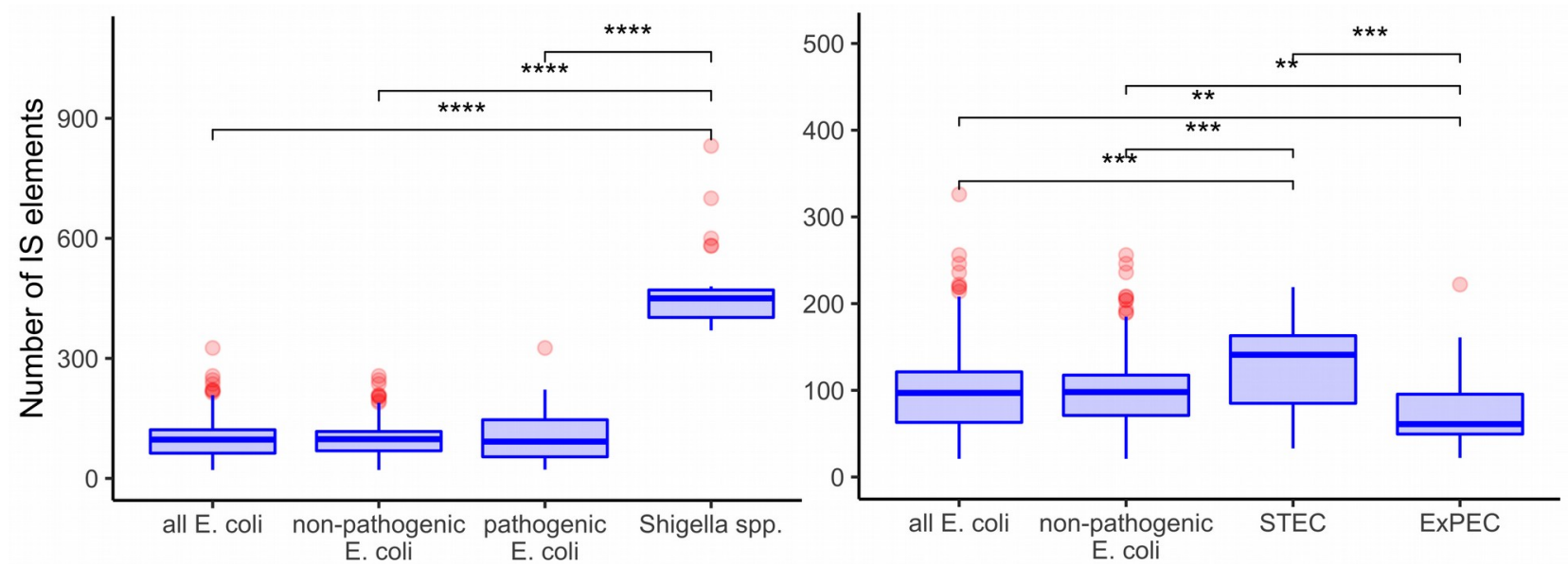

Figure 2. Comparison of IS numbers in (a) Shigella spp., non-pathogenic E. coli, other pathogenic E. coli, and all E. coli (excluding Shigella spp); (b) two most abundant pathovars ExPEC and STEC, non-pathogenic E. coli, and all E. coli (excluding Shigella spp). The WilcoxonMann-Whitney test with the Bonferroni correction: $\left(^{*}\right) p \leq 0.05,(* *) p \leq 0.01$; (***) $p \leq$ $0.001 ;(* * * *) p \leq 0.0001$.

a)

Tree scale: 0.01

Bars scale: 50 IS

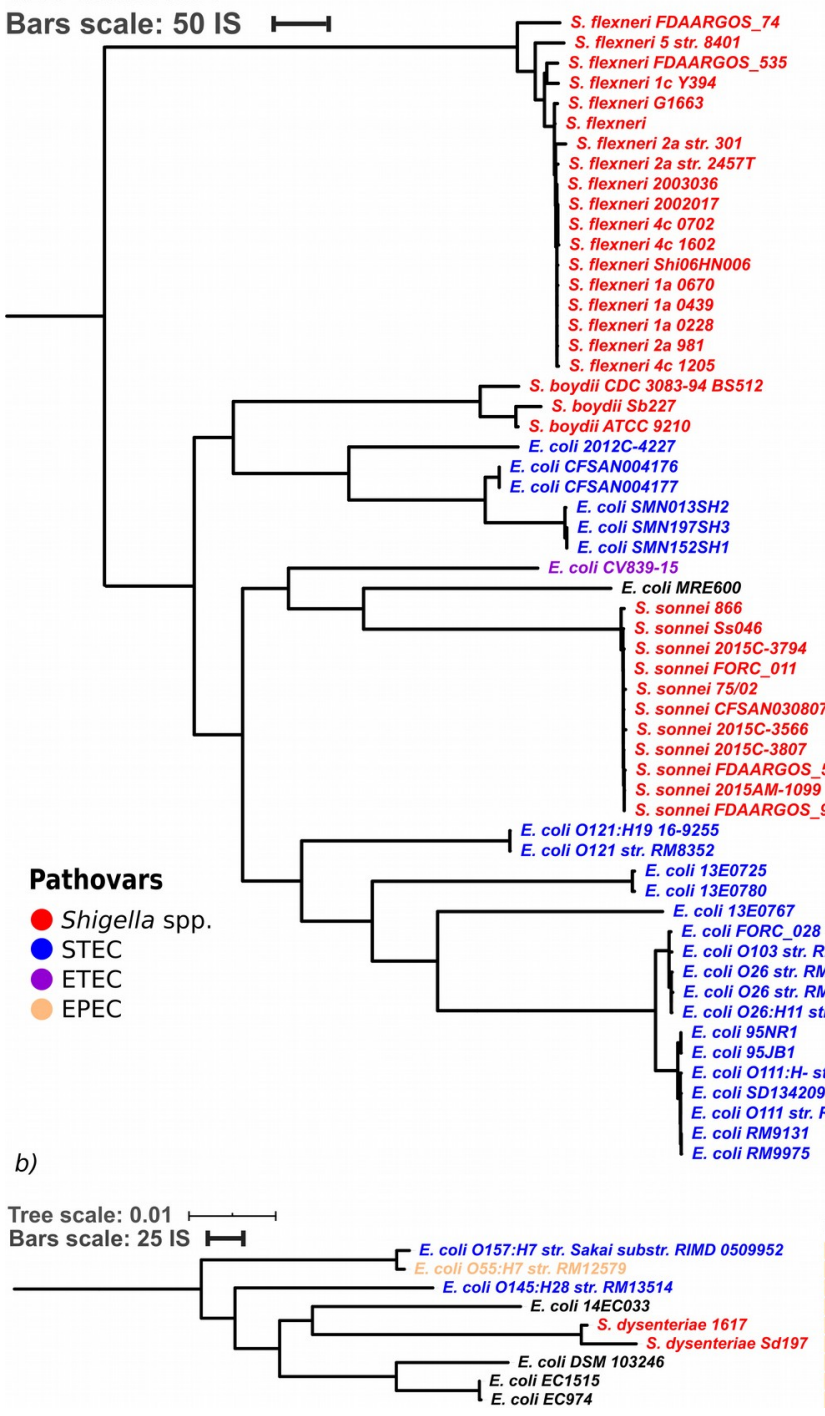

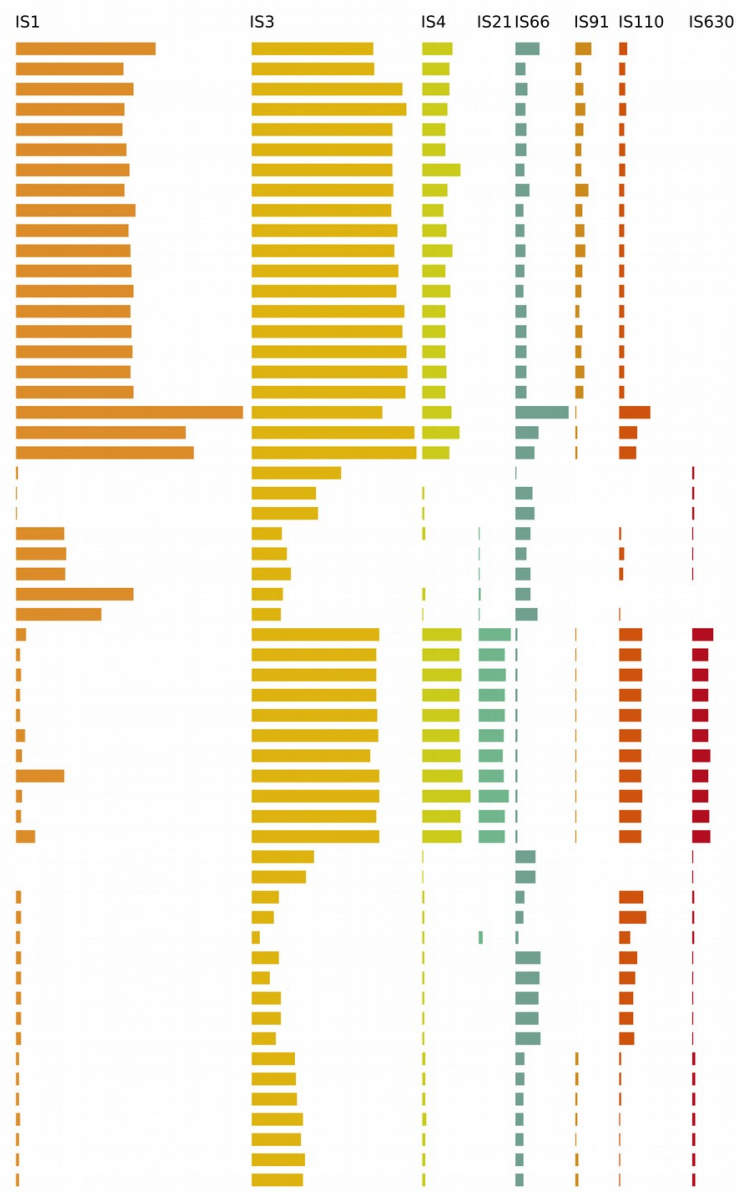

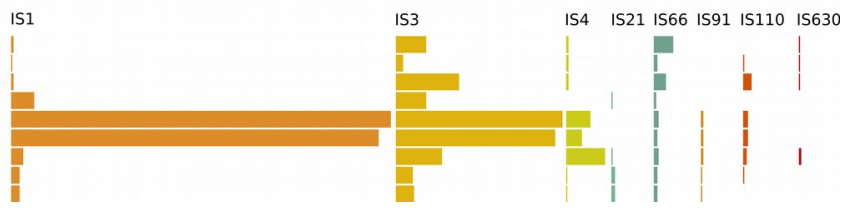


Figure 3. Distribution of IS families in (a) a part of phylogroup B1 and (b) phylogroup $E$. The bars indicate the number of ISs in each family. The trees are also available online (iTOL):

a) https://itol.embl.de/tree/8589127100479001583084903\#,

b) https://itol.embl.de/tree/8589127100208261560504457\#.
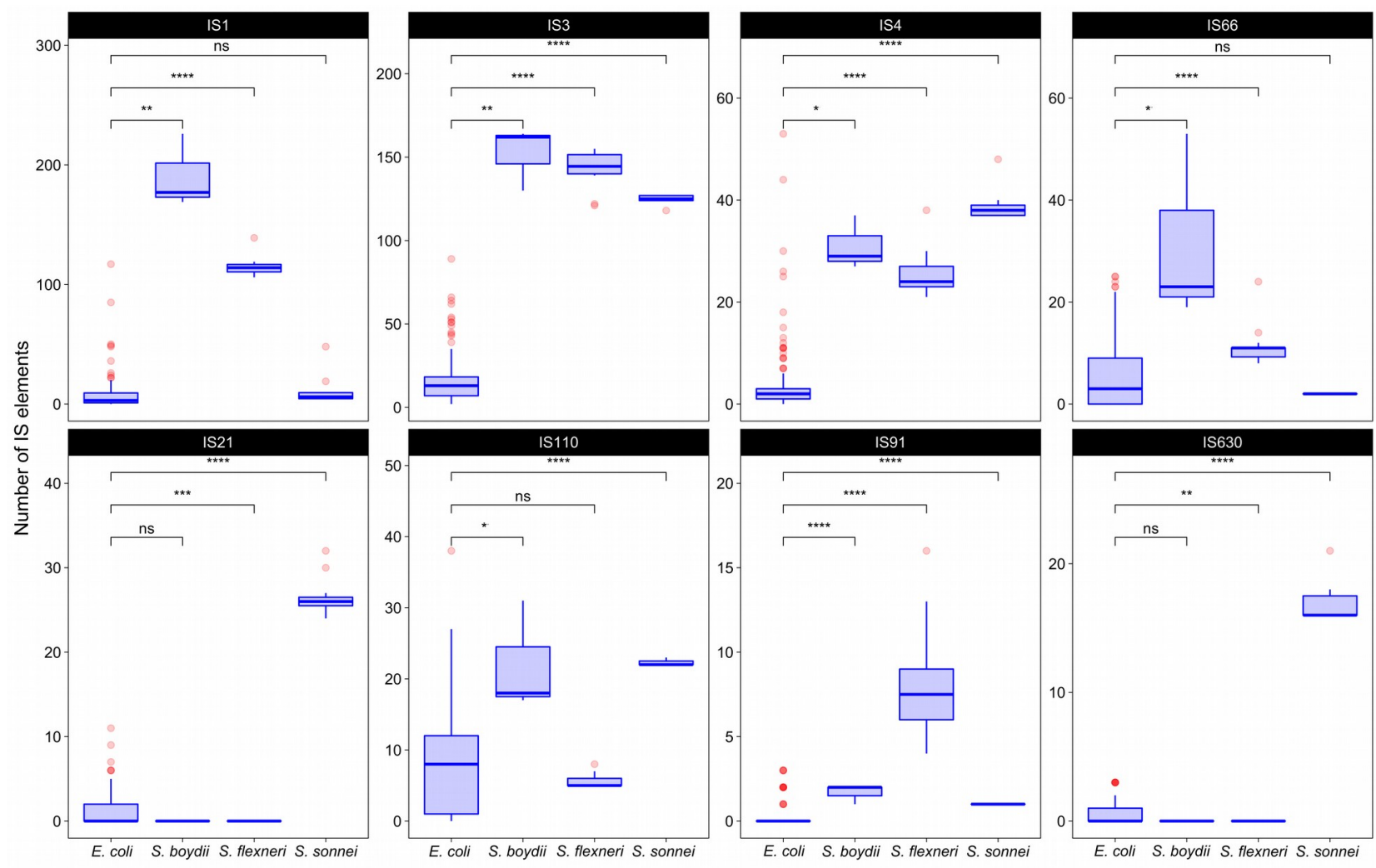

Figure 4. The comparison of selected IS families in Shigella and $E$. coli strains from phylogroup B1. The Wilcoxon-Mann-Whitney test with the Bonferroni correction: $\left(^{*}\right) p \leq 0.05$, $\left.{ }^{* *}\right) p \leq 0.01 ;\left({ }^{* * *}\right) p \leq 0.001 ;(* * * *) p \leq 0.0001$. 


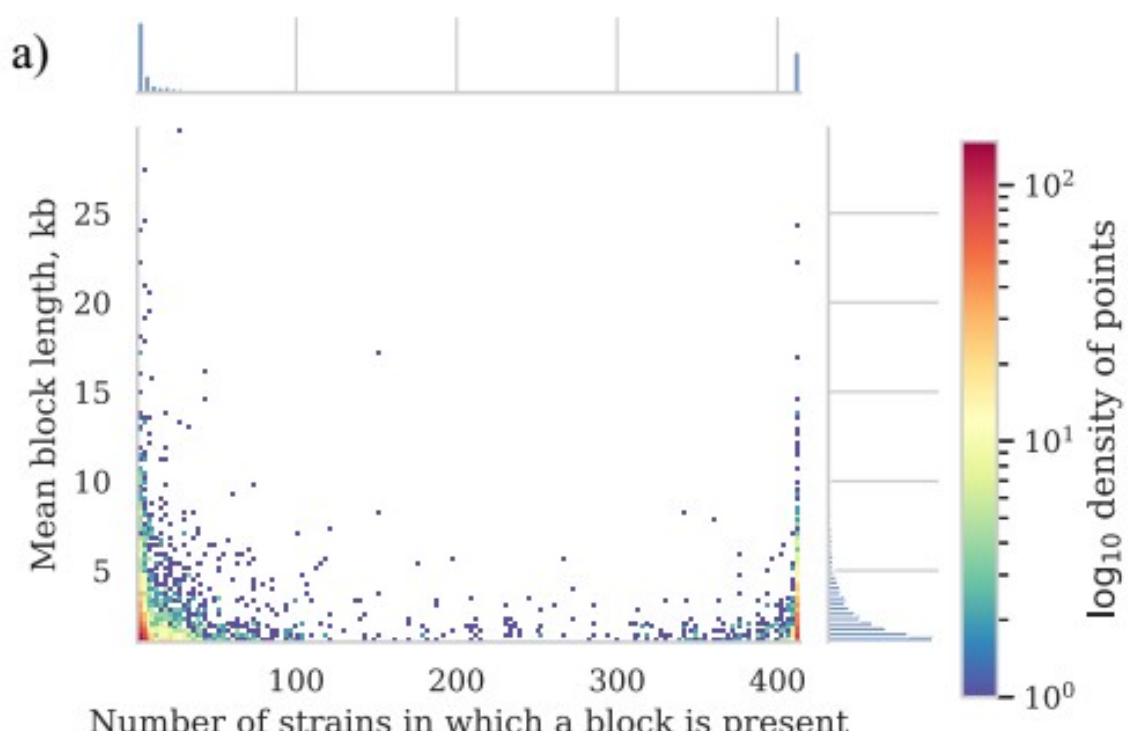

b)

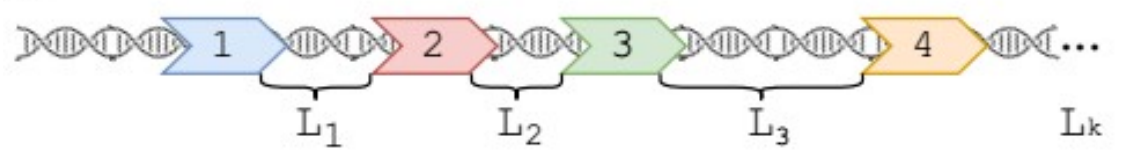

Distribution of the adjacencies' lengths

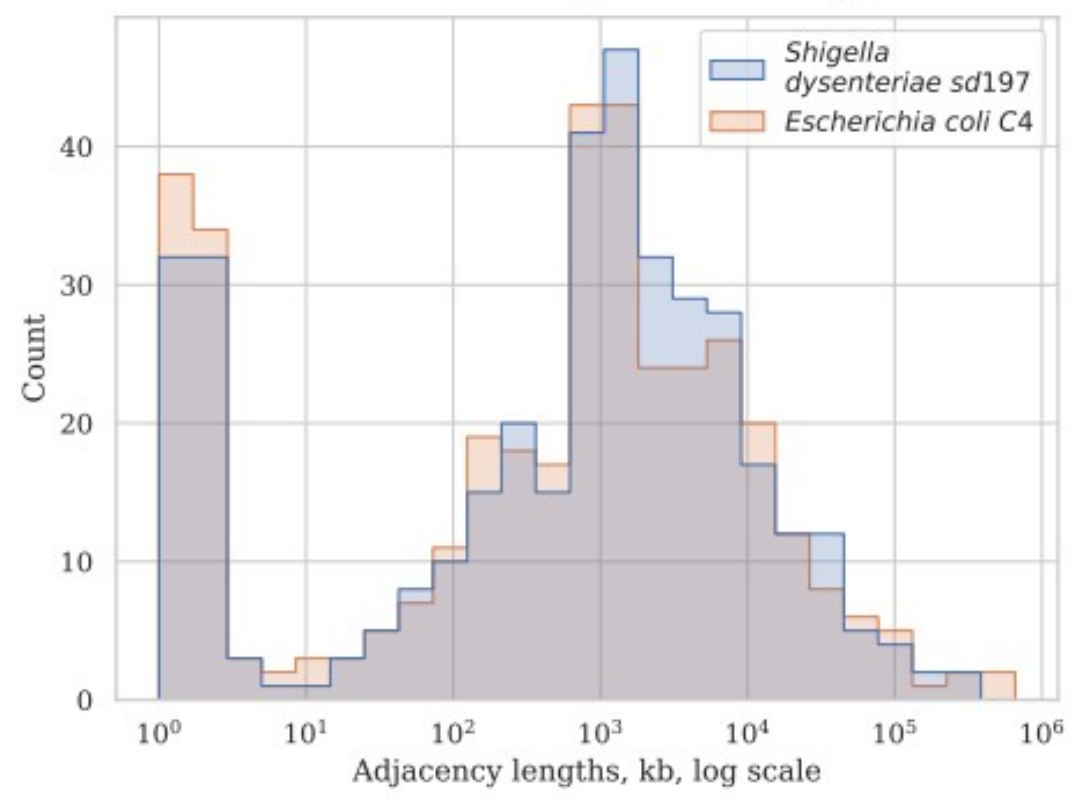

Figure 5. Synteny blocks (a) 2D histogram shows the relationship between the mean block length and the number of strains in which it is present. Color of the point corresponds to the number of synteny blocks with these parameters. $X$-axis histogram shows the frequency distribution of the synteny blocks; $y$-axis histogram shows the length distribution of the synteny blocks (b) Distribution of the adjacencies' lengths in E. coli and Shigella, in nucleotides. Adjacencies are defined as the chromosome regions between the neighboring universal synteny blocks (see Methods). 

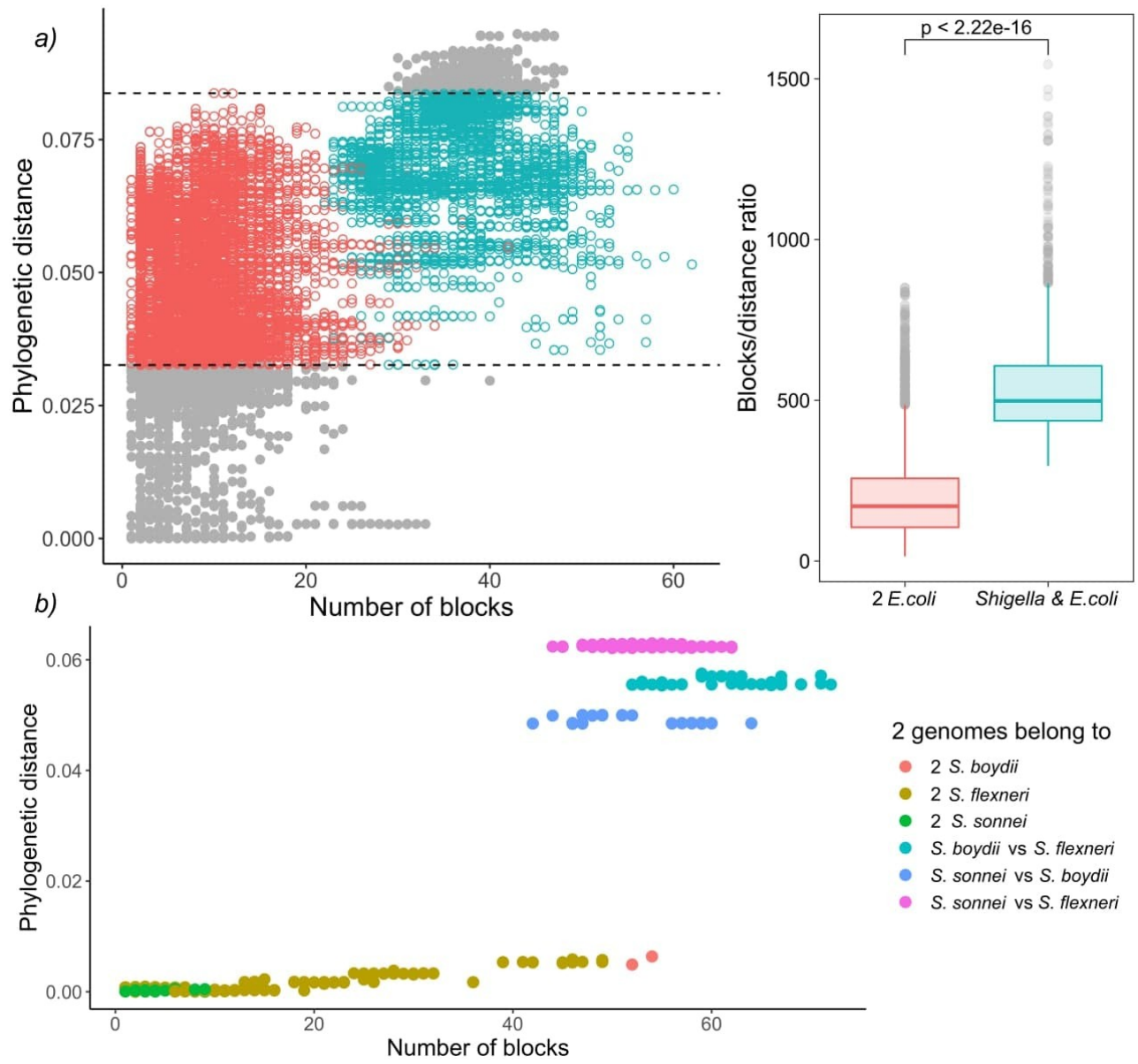

2 genomes belong to

- 2 S. boydii

- 2 s. flexneri

- 2 S. sonnei

S. boydii vs S. flexneri

- S. sonnei vs S. boydii

S. sonnei vs S. flexneri

Figure 6. Comparison of the ratio of phylogenetic distance to the number of synteny blocks for two clusters from phylogroup B1. Only genome pairs in the intersection were compared. The Wilcoxon-Mann-Whitney test $p$-value is given.

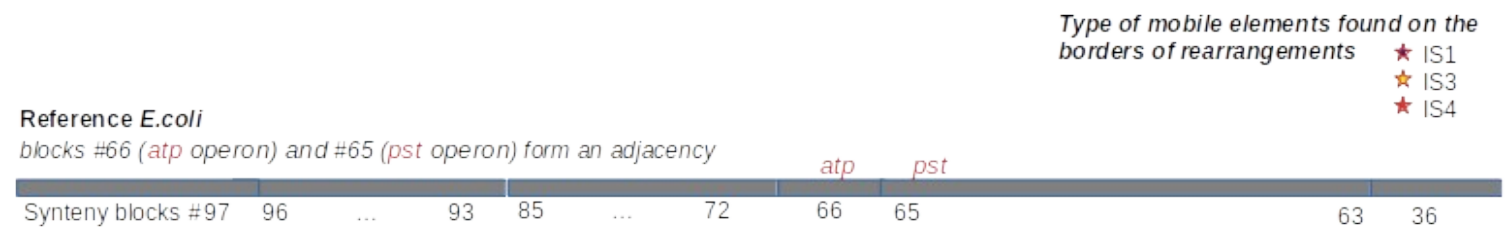

Inversions

E. coll VR50 (inversion of block $\# 66$ )

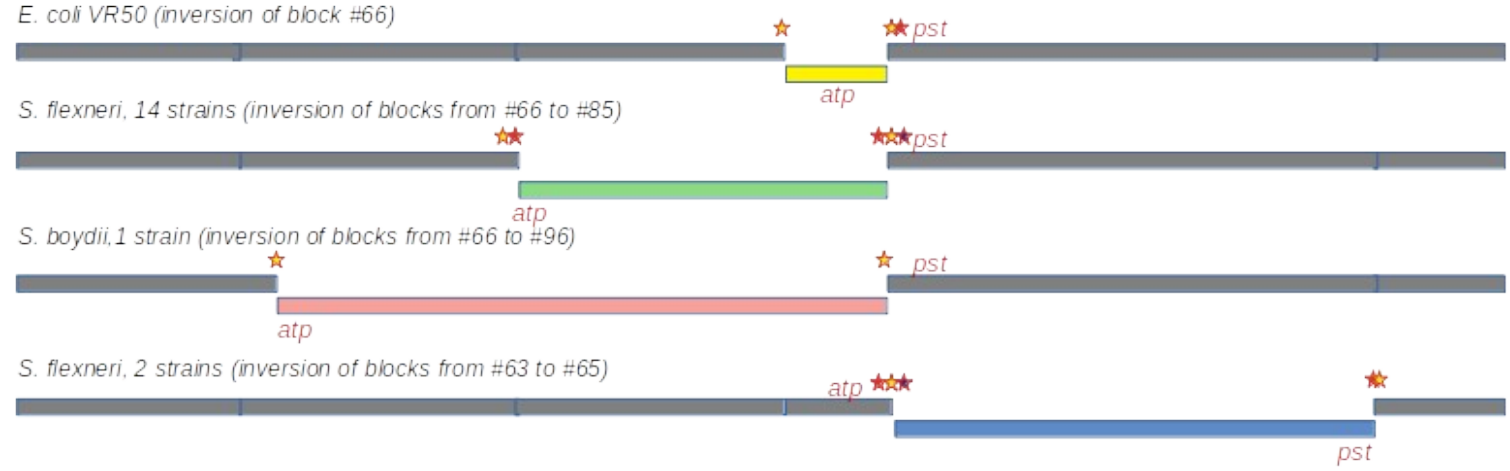

Fig. 7 An example of independent disruption of the adjacency between synteny blocks \#65 contained pst operon (high affinity phosphate transport system) and synteny blocks \#66 contained atp operon (proton-translocating ATPase) by four different inversions. IS families found in the adjacency and putatively responsible for inversions are marked by colored stars. 


\section{Shigella-specific insertions}

a)

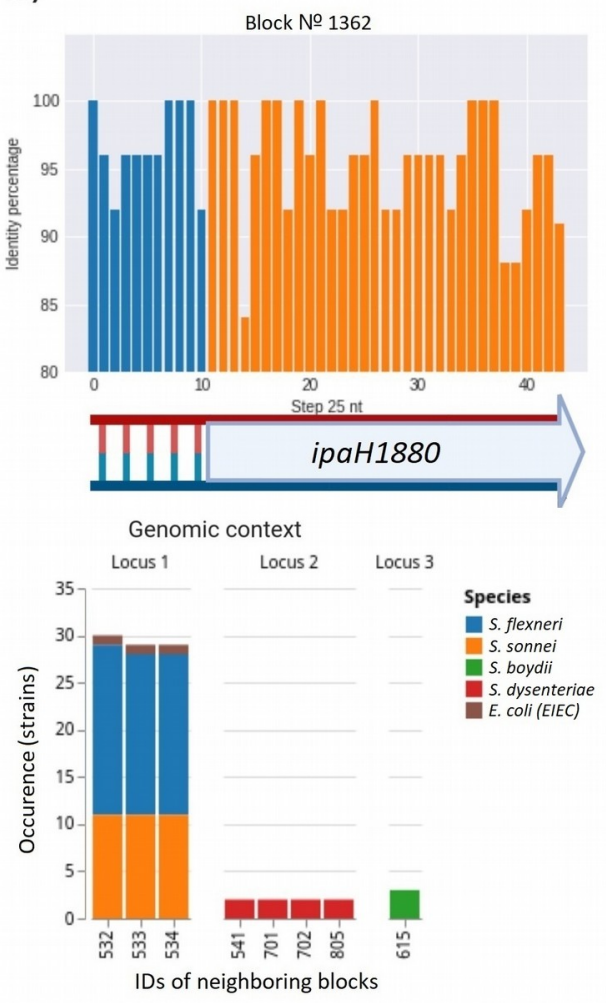

b)

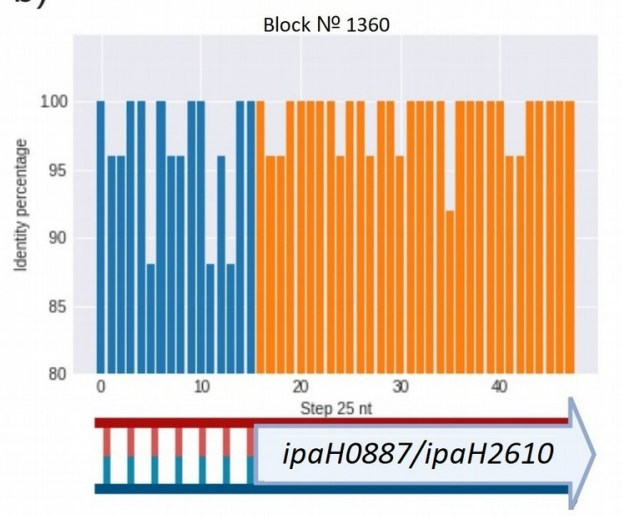

Genomic context

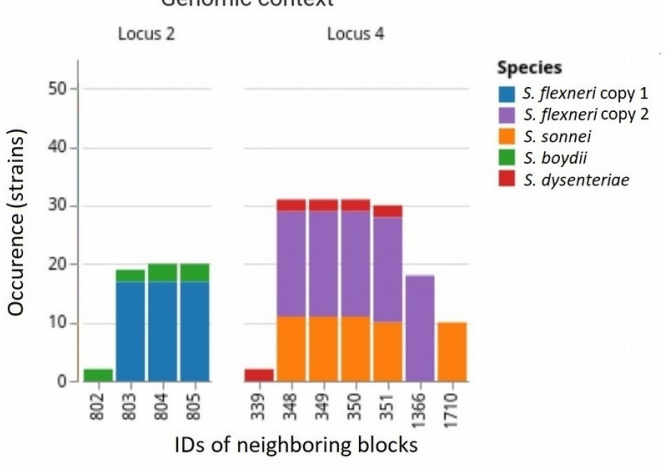

Figure 8. Two parallel Shigella-specific insertions. Both inserted blocks contain long noncoding regions with more than $85 \%$ identity and $i p a H$ genes coding E-3 ubiquitin-ligases with different substrate-specific domains. Identity was calculated for nucleotide alignments of the synteny blocks separately with step $25 \mathrm{nt}$. Location of the inserted blocks in different genomic contexts confirms independent acquisition of the block by different Shigella lineages; in S. dysenteriae block \#1360 was inserted twice in different loci. We took into consideration up to five of the nearest blocks, both in upstream and downstream direction, at the distance not exceeding $10 \mathrm{~kb}$. Colors correspond to Shigella lineages. Gene composition of the neighboring blocks for both insertions is shown in Supplementary Table S5.

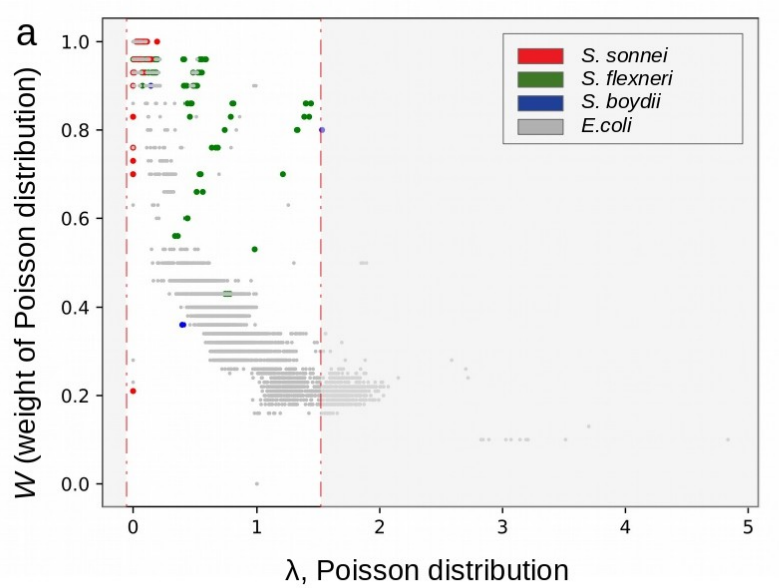

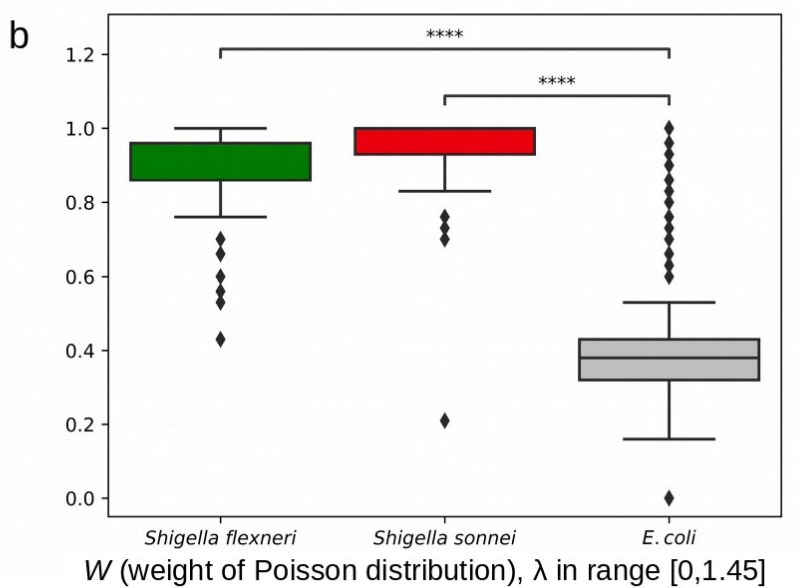


Figure 9. Rates of homologous recombination. (a) Fractions of horizontally transferred fragments in strains from the B1 phylogroup calculated as the weight of the Poisson distribution (see Methods). (b) The weight of Poisson distribution in Shigella and E. coli in the same interval of evolutionary distances between strains.

Figure S1. Phylogenetic trees. (a) E. coli phylogenetic tree. The tree is based on the nucleotide alignment of universal single-copy orthologs. Strains shown in red were excluded from further analysis. (b) E. coli/Shigella phylogenetic tree. The tree is based on the nucleotide alignment of universal single-copy orthologs with $95 \%$ identity threshold.

Figure S2. Construction of breakpoint graph. (a) Genome graphs of unichromosomal circular genomes $P=(0,1,2,3,4,5)$ and $Q=(0,1,-4,-3,-2,5)$, the adjacency edges of the genome $P$ (left) are shown in blue, the edges of the genome $G$ (right) are shown in red (b) The breakpoint graph $G(P, Q)$ of genomes $P$ and $Q$ (c) The multiple breakpoint graph of five unichromosomal circular genomes.

Figure S3. Chromosome maps (a) Escherichia coli C4 (b) Escherichia coli cfsan029787 (c) Shigella flexneri 1a 228 (d) Shigella boydii atcc 9210 (e) Shigella dysenteriae sd197 (f) Shigella sonnei fdaargos 524 . The inner circle - GC-skew, the second (blue) circle - synteny blocks, the third (green) circle - universal synteny blocks, the fourth (grey) circle — ISs, the fifth (grey) circle - density of ISs.

Figure S4. Phylogenetic distance versus the number of synteny blocks for each pair of genomes (a) in phylogroup A, (b) in phylogroup B2, (c) in phylogroup D, (d) in phylogroup F. Each point represents a pair of genomes and is coloured according to the genomes the pair includes.

Figure S5. Inversion events reconstructed by MGRA software. (a) A cladogram with numbers of inversions shown for each branch. (b) The corresponding phylogenetic tree. Phylogroups are marked with coloured strips, pathogenic strains are shown in different colours. Both trees are unrooted. The trees are also available online (iTOL): https://itol.embl.de/tree/9318063252480721583313649.

Figure S6. Genome sizes in (a) Shigella, non-pathogenic E. coli, other pathogenic E. coli and all E. coli (excluding Shigella spp); (b) two most abundant pathovars - ExPEC and STEC, nonpathogenic E. coli and all E. coli (excluding Shigella spp). (c) different groups of E. coli. The numbers above boxplots indicate the number of genomes in each group. The Wilcoxon-MannWhitney test with the Bonferroni correction: $\left(^{*}\right) p \leq 0.05,\left({ }^{* *}\right) p \leq 0.01$; (***) $p \leq 0.001$; $(* * * *) p \leq 0.0001$.

Table S1. The list of E. coli and Shigella genomes included in the analyses.

Table S2. Coordinates of synteny blocks.

Table S3. Adjacencies of common synteny blocks.

Table S4. Copy number of non-common synteny blocks.

Table S5. Gene composition of synteny blocks surrounding the Shigella-specific insertions. 